American Journal of Economics and Business Administration 3 (1): 18-23, 2011

ISSN 1945-5488

(C) 2010 Science Publications

\title{
A Virtual Repository Approach to Departmental Information Sharing
}

\author{
Zulikha Jamaludin and Wan Hussain Wan Ishak \\ Department of Computer Science, College of Arts and Science, \\ University Utara Malaysia, 06010 Sintok, Malaysia
}

\begin{abstract}
Problem Statement: Realizing the difficulties in information sharing among the academicians, this study introduces an alternative information sharing model supporting information management features. Objective: The model is designed in an attempt to facilitate information distribution and information sharing at minimum effort and cost. Approach: A centralized database approach is used in the model enabling any educator in the higher learning institution to participate and manage the database, conceptualizing that the database is their very own 'personal library'. This model is then implemented in an application given a persona name ViRepo-a web-based internal virtual repository. The process of analyzing, designing and implementing the model into ViRepo is sufficiently reported in this study. Results: ViRepo proved that the model, in actual fact, allows information access, information sharing, information management, partnership enhancement and allows an utmost repository control by each and every participating educator. Also, each user is able to obtain a better picture about the overall collection's field, possession status and type (printed or electronic), which in turns reflects the owners' interest and field of research. Conclusion: Being relatively simple in terms of architecture, ViRepo is highly potential to be implemented in learning organization as it can enrich the knowledge of the users and owners and at the same time reduce the cost of purchasing reading materials.
\end{abstract}

Key words: Virtual repository, ViRepo prototype, information sharing, priority ratings, database approach

\section{INTRODUCTION}

Every educator in schools or universities possess collections of books, journals, articles, proceedings, theses and other documents. They can be in printed or electronic form. These documents are used for Teaching and Learning (T\&L) as well as Research and Development (R\&D) activities. It is fair to said that they actually own a personal library in their study space or in their office. However, the information regarding the collection in their 'very own library' is not recorded anywhere, making it extremely difficult to find out what they have/do not have in their collection, let alone to share the information or the material among them.

Studies carried out by Welsh $(1987 ; 2002)$ verified that educators are more than willing to share their collection but are not able to do so due to the short of appropriate platform for such sharing. As a consequence, it is not easy to find information regarding books, journals, magazines or other documents especially when we are in a dire need for a particular information and we are sure that someone in the department has it. It is just that we are not sure who has it. Also, it is surely not practical to call each and every lecturers in the department to find out!

In our 15-year experience working as lecturers in universities, we found that it is taxing to find and share information relating to $T \& L$ and $R \& D$ materials which are stored in the educator's collection because no one knows for sure what type of materials are in their colleague's collection. In some cases, even the educator himself is unaware of what his collection contains (Welsh, 2002; Paganelli and Pettenati, 2005). This condition is a hindrance to information sharing, which could subsequently create a barrier for collaboration in related fields.

Motivations and objectives: The fact that information sharing is one of the major problem among educators (Welsh, 1987; 2002; Eckel, 2007), we propose an alternative model for information and knowledge sharing in this study. The model involves educators in a university as subjects and the test domain respectively. Based on the model we further design and implement

Corresponding Author: Zulikha Jamaludin, Department of Computer Science, College of Arts and Science, University Utara Malaysia, 06010 Sintok, Malaysia 
ViRepo, a prototype of a virtual library that support information sharing and collaboration, specifically for identifying and locating materials that was previously 'hidden' in the lectures' room.

In particular, we target to (1) compare and analyze a few architecture of virtual libraries known to be efficient, i.e., able to perform its main function at a reasonable speed and minimum effort; (2) create an active information sharing model within the scope of a virtual library; (3) incorporate typical features of a virtual library into the model created; (4) collect collection information from lecturers in one of the faculty (Faculty of Information Technology - FTM) in University Utara Malaysia (UUM); (5) design a centralized database to store information about the collection owns lecturers in FTM; (6) design an engine for the small scale virtual repository prototype using the architecture, model, typical features, document information and the database as predetermined in objective 1-5 above; (7) build a user interface for the said prototype.

The scope and benefit of the study: Objectives 1-7 above was achieved within the scope of computer networking in FTM that is utilized by the lecturers involved in this study. As for the scope of the database, we limit the record size of 100,000 records. We assume the amount is adequate since FTM has 80 active staff and it is estimated that each staff on average possess 1000 items. Our information sharing model is based on a unit database model, assuming a lecturer as a unit and the department as the whole community. As for the prototype, it is an on-line web-based application equipped with a web-engine to process data from the information provider (the lecturers who are also the owner of the collection), the webmaster (the administrator) and users (all other lecturers who perform the search).

We propose this model in an attempt to save effort and cost of information sharing. This model is unique as it involves the development of a virtual repository where lecturers become the contributor, the user, as well as the owner of the repository. Such a repository enables a systematic information sharing as long as each user keeps the database updated.

A lecturer can become a participant, a manager and also an owner for the virtual library which in turn offer another benefit in terms of information access, sharing and management. Also, each user will obtain a better picture about the overall collection's field, possession status and type (printed or electronic), which in turns reflects the lecturer's interest and field of research. This information contributes towards generating a better network between senior and junior lecturers, increase collaboration and teamwork. At the same time, the owner still maintain full control and possession towards their collection in their study space/room. Furthermore, this model can serve as a repository of learning resources (Ghaleb et al., 2006), which can support e-learning activities. In particular, using Service Oriented Architecture (SOA) this model has a potential to be integrated with Learning Management System (LMS). Jabr and Omari (2010) have demonstrated the use of SOA to integrate various applications on World Wide Web into their LMS.

Related studies: A virtual repository is normally built to store research material collected by a certain webmaster. The webmasters involved as agents who filter and categorize information to be stored in a certain virtual repository ( United States Department of Justice, 2010, Paganelli and Pettenati, 2005). Obviously there are many virtual library such as those listed in the Srivinas (2010). However, these libraries are the 'solecontrol' kind of library, i.e., owned and managed by the designer and the webmaster if it is the on-line type of library. The control is not shifted to the owner of the collection but stay put on the owner of the repository. We regard this model as outdated because it is authoritative, making the owner of the collection left without any hold over their own collection. The owner are regarded as having the same status as the ordinary users (viewer), where they can only perform routine activities of searching, checking, scheduling, sorting and printing without any 'power' to edit, add, delete and other activities that involve changing the entry in the database.

However, their model for information design (called GDRC model) is useful because it also answer similar questions that we are trying to address namely, what is the main message we want to share? Which medium we want to use? What resource are we to use? Who are the main end-users? How can information be presented for this purpose? And how does the model on information design facilitate this? (Srivinas, 2010).

We also consider (www.wikipedia.com) as one of the different model to be compared with. Wiki's model, however, does not give each and every user a similar vision in terms of structure and types of content. Without something that can push the structure as a focus, then it will not support the development of users' mental and conceptual model, which in turn will cause difficulty in performing functions available within Wiki. However, it is still too early to compare the ViRepo model with Wiki because of the size of records within Wiki and ViRepo is extremely different. Wiki's database is gigantic in terms of volume, whereas ViRepo is still small. Certain models serve best for 
small database, while some others serve efficiently with large database and not so useful for small database.

\section{MATERIALS AND METHODS}

Model development method: Three repository models are compared in order to decide on ViRepo model. We compare and examine hermeneutically the following models: Honeybee information sharing model (Walker, 2003), Paganelli and Pettenati (2005) model and Centralised information repository model (United States Department of Justice, 2010). The elements compared and analyzed are the entities involved, the requirements for mandatory functions, the actual functions readily available in each of the repository's models.

The entities identified are administrators, contributors and users. In all cases, contributors are also users. As for the functional requirements, our Hermeneutic study enables us to identify the 15 functions being implemented in various repositories. However not all of toes functions are widely used. There are only seven popular functions that have been used by all repositories available on-line. Table 1 list down the functions and mark those that are popular.

Based on the finding, we decided to implement only those seven popular functions in our model. Those function are operable by three entities namely the webmaster (the administrator), the owner (the contributors with full control on their collection) and users (other than contributors, with limited control on the collection). The users could, at the same time, be the owner. These users have full control on their own collection but limited control on others. Table 2 shows the summary of entities and functionality decided for ViRepo.

This repository is termed as faculty's repository by the webmaster/administrator, termed as personal library by the owner of the collection and termed as virtual repository by the users who do not own any material in the collection.

Figure 1 illustrates the context diagram showing entities, functions and requirements as listed in Table 2. The diagram also shows the interaction between ViRepo and those entities.

Owners and users have login, password and their identification. Each item in the collection has its owner, types, authors, title, ISBN/ISSN (for books and journals), year published and other notes that can be edited and updated by the owner.

ViRepo development process: Figure 2 shows the summarized process of building ViRepo. The process, in general follows the rapid prototyping method.

There are seven main activities carried out in developing the ViRepo prototype. Firstly, selecting functions to be implemented in ViRepo. We

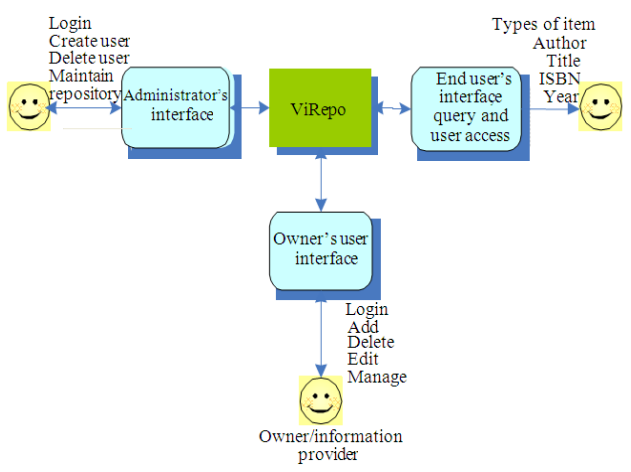

Fig. 1: The context diagram showing the relationship between ViRepo with its three main entities

Table 1: Functions and popular functions in virtual repository

\begin{tabular}{l}
\hline Functions \\
Create the collection (by creating a new category \\
for documents) \\
Manage the collection (add, update, delete) \\
Create contributors' records \\
Manage contributors' information (add, update, delete) \\
Manage bibliographic information (add, update, delete) \\
Perform search \\
Keyword search \\
Natural language search \\
Keyword and Boolean operator search \\
Advanced search \\
Field-based search \\
Search within a search result \\
Manage search results \\
$\begin{array}{l}\text { Sort according to documents' suitability } \\
\text { Sort a certain field }\end{array}$ \\
Filter or categorize documents according to category \\
Fix the size/number of the display screen \\
Build a full display and display the same document \\
from different contributors \\
Build a summary display \\
Download documents by providing links to the \\
on-line documents \\
Generate documents in pdf format \\
Give scores and comments towards a document \\
Login and logout system \\
Make printouts (some provides special printing formats) \\
Record and count document access
\end{tabular}

Record and count document access

use priority ratings to select the functions. Secondly, developing the database using the Rapid Application Development (RAD) approach and guidance (Elmasri and Navathe, 2003). Thirdly, developing the database processing engine and the web-based search engine to access information in the database. Both engines forms the prototype's engine. This step is also carried out using RAD method. Fourthly, designing the user interface to enable the interaction between the engine and the administrator, owners and users. Firstly, collecting data from the owners periodically using email push. Next, designing the repository and restructuring all collections in the repository. Finally, integrating the database, prototype engine and the user interface to develop a complete repository termed as ViRepo prototype. 
Am. J. of Economics and Business Administration 3 (1): 18-23, 2011

Table 2: Entity, functions and requirements for the model

\begin{tabular}{lll}
\hline Entity & Function & Requirement for function access and sharing \\
\hline 1. Administrator/webmaster & Create contributors' records & \\
& Add contributors' records (when necessary) & \\
& Delete contributors' records (when necessary) & \\
& Other editing (on demand or when necessary) & \\
& Manage contributors' record (on demand or when necessary) & \\
& Maintain the repository + all user function & Login and password \\
& & \\
2. Owner (lecturers who put up & Add collection & \\
material for his/her & Delete collection & \\
own collection) & Search collection, owner & Login and password \\
Edit collection & Change password & \\
& Manage own library + all user function & \\
3. User (lecturers who are not & Search collection, owner & \\
owner) & Print \\
& Copy & Login and password \\
\hline
\end{tabular}

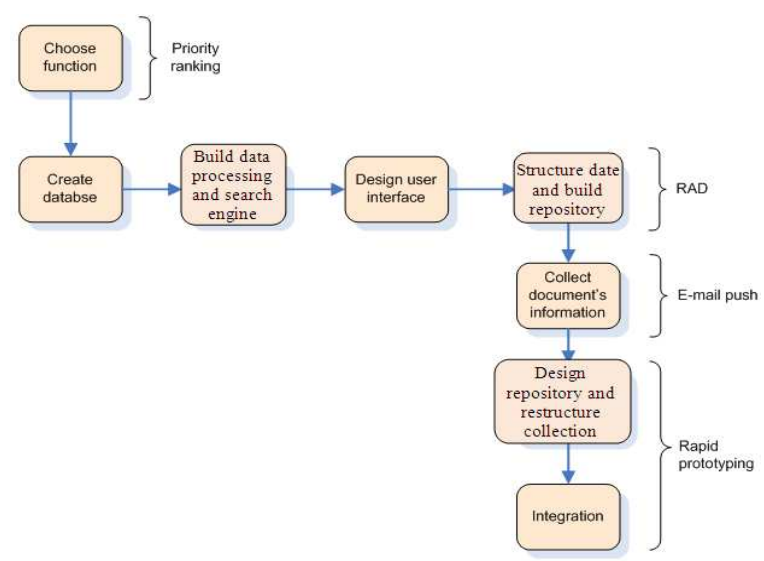

Fig. 2: The process for building the ViRepo prototype

\section{RESULTS}

The result of the study is in the form of information sharing model and ViRepo prototype. Figure 3 depicts the said active information sharing model which we use as a basis for developing the FTM's virtual library prototype.

In realizing the model into the prototype, we begin with designing the ViRepo architecture and components. The components required are a server, a database, a user interface, a web server and a browser. The connection from the database to the web server are established through Active Server Page (ASP) scripts, whereas the connection from the user interface to the browser is accomplished via hypertext transfer protocol (http). The architecture and the related components of ViRepo is presented in Fig. 4.

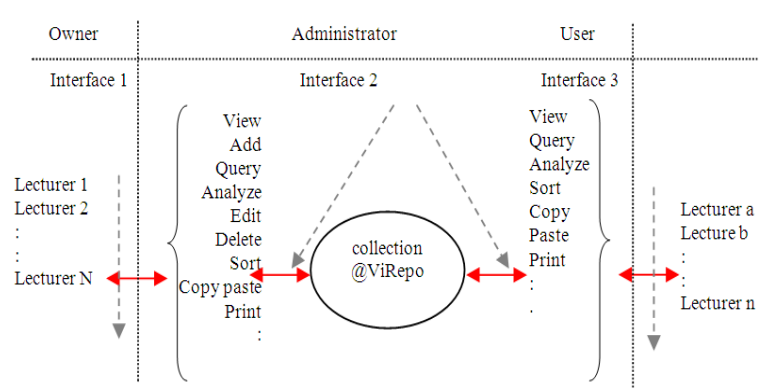

Fig. 3: The active sharing model for ViRepo

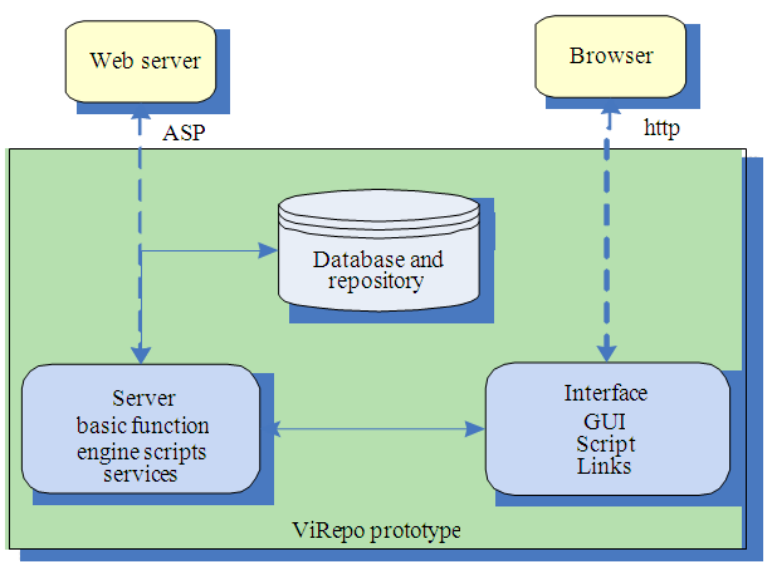

Fig. 4: Components in the ViRepo architecture

The implementation of ViRepo architecture and component yields the desired prototype. Figure 5 depicts one of the ViRepo displays, viewed in a user interface meant for owners. 
Am. J. of Economics and Business Administration 3 (1): 18-23, 2011

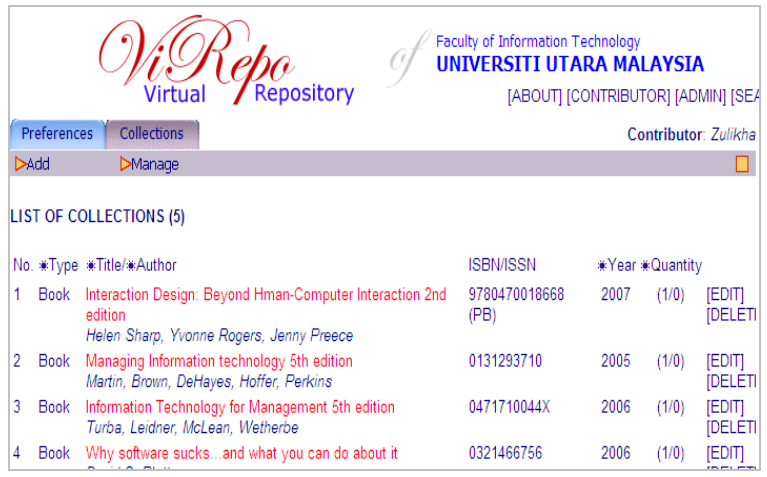

Fig. 5: One of the ViRepo displays (a user interface for submenu collection $\rightarrow$ manage)

This prototype is being tested by academic staff of FTM for reliability and robustness. Subsequently the usability testing will also be carried out.

\section{DISCUSSION}

The model we based our virtual repository development is proven to be efficient despite being quite simple. Our objective (1 to 7) are accomplished by implementing the active sharing model for ViRepo. Being simple and small in terms of database size $(100,000$ records), ViRep is unique in terms of e-mail push feature whenever a material is ready to be shared. All the owner need to do is mark the related check box, and an e-mel will be sent out to users who requested the material. Compared to other virtual repository, this feature is unique only to ViRepo. The usefulness of this feature is yet to be studied because the usability testing for ViRepo is not yet done. It is therefore recommended that ViRepo should undergo usability evaluation, using a proper usability tool such as Tobii Studio.

Virtual libraries utilizing centralized repository model such as those reported by Srivinas (2010) and United States Department of Justice (2010) are rich in terms of security feature. ViRepo is very much in short on this. In fact at this stage we have not implemented any security features other than password and login. Security feature for ViRepo is another recommended future study.

\section{CONCLUSION}

Formerly, information regarding academic references that are available in a lecturer's study space/room are not recorded anywhere, until it becomes very difficult for other academics to find out what one lecturer posess. We hope that with the realization of
ViRepo, it would assist in information sharing at least for books, journals, articles, proceedings and theses in printed or electronic form. Such information sharing is crucial to support $\mathrm{T} \& \mathrm{~L}$ as well as $\mathrm{R}$ and $\mathrm{D}$ activities in any higher learning institutions, or even schools.

This study propose an information sharing model and using the model we also develop a prototype, ViRepo, as a means to verify the model, or as a prove of concept. The process of formulating the model and designing the prototype begin with examining a few efficient virtual libraries' architecture, instilling typical features $o$ fan effective virtual library, collecting information on collection items from lecturers, building a centralized database, desiggining and developing the prototype's engine and crafting the user interface. The resulting prototype is a web-based on-line application equipts with engines to process data from users, owners and the administrator.

Such a repository model benefits us in terms of accessing, sharing and managing information in lecturers' collection. Participating in ViRepo opens up another opportunity for generating network among lecturers, which in turns upsurge collaboration dan teamwork.

In theory, the ViRepo is considered successful. Nevertheless, its architecture needs further investigations. We need to examine the ViRepo performance when the size of the database exceeds 500,000 records. Such study is required in order to determine the reliability of the prototype. The same goes with the Interaction Design (IxD) of ViRepo. Its IxD needs to be customized for the target user if it is to be implemented in another faculty or another university or schools, in order to accommodate the prototype with the user profile in those places.

By modeling and implementing ViRepo, we believe that it would be easy for the department to expand this design into an institutional repository. The repository would in turn, serve as one of the entity under Central Research Information System (CRIS). The ability to categories, sort and group items according to types, researchers, fields of research and team members would greatly assist other researchers in their study.

\section{REFERENCES}

Eckel, B., 2007. We need a new information-sharing model for the internet. Comput. Thoughts. 2005 http://www.artima.com/weblogs/viewpost.jsp?threa $\mathrm{d}=113330$ 
Elmasri, R. and S.B. Navathe, 2003. Fundamentals of Database Systems. 4th Edn., Addison Wesley, ISBN: 978-0321122261, pp: 1009.

Ghaleb, F.F.M., S.S. Daoud, A.M. Hasna, J.M. Jaam and H.F. El-Sofany, 2006. A web-based e-learning system using semantic web framework. J. Comput. Sci., 2: 619-626. DOI: 10.3844/jcssp.2006.619.626

Jabr, M.A. and H.K.A. Omari, 2010. E-learning management system using service oriented architecture. J. Comput. Sci., 6: 285-295. DOI: 10.3844/jcssp.2010.285.295

Paganelli, F. and M.C. Pettenati, 2005. A model driven method for the design and deployment of webbased document management systems. J. Digital Inform., 6: http://journals.tdl.org/jodi/article/viewArticle/67/149

Srivinas, H., 2010. WWW Virtual Library: Information Design and management. The Global Development Research Center. http://www.gdrc.org/info-design/
United States Department of Justice, 2010. (10. Centralised information repository model http://it.ojp.gov/documents/asp/models/section3.ht m. Retrieved 12 March, 2010

Walker, R.L., 2003. Emulating the honeybee information sharing model. Proceedings of International Conference on Integration of Knowledge Intensive Multi-Agent Systems, 30 Sep. $\quad-4$ Oct. pp: 497-504. DOI: 10.1109/KIMAS.2003.1245091

Welsh, P., 1987. Are administrators ready to share decision making with teachers. Am. Edu., 11: 23-48. www.eric.ed.gov/ERICWebPortal/recordDetail?ac cno $=$ EJ351824

Welsh, P., 2002. Opening minds: Why i teach. Am. Edu., 26: 7-9. http://www.aft.org/newspubs/periodicals/ae/fall200 2/welsh.cfm 